\title{
SOME RESULTS CONCERNING THE $p$-ROYDEN AND $p$-HARMONIC BOUNDARIES OF A GRAPH OF BOUNDED DEGREE
}

\author{
Michael J. Puls \\ CUNY, John Jay College, Department of Mathematics \\ 445 West 59th Street, New York, NY 10019, U.S.A.; mpuls@jjay.cuny.edu
}

\begin{abstract}
Let $p$ be a real number greater than one and let $\Gamma$ be a connected graph of bounded degree. We show that the $p$-Royden boundary of $\Gamma$ with the $p$-harmonic boundary removed is an $F_{\sigma}$-set. We also characterize the $p$-harmonic boundary of $\Gamma$ in terms of the intersection of the extreme points of a certain subset of one-sided infinite paths in $\Gamma$.
\end{abstract}

\section{Introduction}

Let $\Gamma$ be a graph with vertex set $V_{\Gamma}$ and edge set $E_{\Gamma}$. We will write $V$ for $V_{\Gamma}$ and $E$ for $E_{\Gamma}$ if it is clear what graph $\Gamma$ we are working with. For $x \in V$, $\operatorname{deg}(x)$ will denote the number of neighbors of $x$ and $N_{x}$ will be the set of neighbors of $x$. A graph $\Gamma$ is said to be of bounded degree if there exists a positive integer $k$ such that $\operatorname{deg}(x) \leq k$ for every $x \in V$. A path $\gamma$ in $\Gamma$ is a sequence of vertices $x_{1}, x_{2}, \ldots, x_{n}$ where $x_{i+1} \in N_{x_{i}}$ for $1 \leq i \leq n-1$ and $x_{i} \neq x_{j}$ if $i \neq j$. Assume throughout this paper that all infinite paths have no self-intersections. A graph is connected if any two given vertices of the graph are joined by a path. All graphs considered in this paper will be connected, of bounded degree with no self-loops and have countably infinite number of vertices. We shall say that a subset $S$ of $V$ is connected if the subgraph of $\Gamma$ induced by $S$ is connected. The Cayley graph of a finitely generated group is an example of the type of graph that we study in this paper. By assigning length one to each edge of $\Gamma, V$ becomes a metric space with respect to the shortest path metric. We will denote this metric by $d(x, y)$, where $x$ and $y$ are vertices of $\Gamma$. Thus $d(x, y)$ gives the length of the shortest path joining the vertices $x$ and $y$. Finally, if $x \in V$ and $n \in \mathbf{N}$, then $B_{n}(x)$ will denote the metric ball that contains all elements of $V$ that have distance less than $n$ from $x$.

Let $p$ be a real number greater than one. In Section 2 we will define the $p$-Royden boundary of $\Gamma$, which we will indicate by $R_{p}(\Gamma)$. We will also define the $p$-harmonic boundary of $\Gamma$, which is a subset of $R_{p}(\Gamma)$. We will use $\partial_{p}(\Gamma)$ to denote the $p$-harmonic boundary. Our motivation for investigating the $p$-harmonic boundary of a graph is its connection to the vanishing of the first reduced $\ell^{p}$-cohomology space of a finitely generated group. More specifically, this space vanishes if and only if the $p$-harmonic boundary of the group is empty or contains exactly one element, see [6, Section 7] for the details of this fact. Gromov conjectured in [1, page 150] that the first reduced $\ell^{p}$-cohomology space of a finitely generated amenable group vanishes. Thus, a better

doi:10.5186/aasfm.2012.3705

2010 Mathematics Subject Classification: Primary 60J50; Secondary 43A15, 31C45.

Key words: $p$-Royden boundary, $p$-harmonic boundary, $p$-harmonic function, $F_{\sigma}$-set, extreme points of a path, $p$-extremal length of paths. 
understanding of the $p$-harmonic boundary could be helpful in resolving Gromov's conjecture.

Recall that in a topological space a set is said to be $F_{\sigma}$ if it is a countable union of closed sets. In this paper we will prove that $R_{p}(\Gamma) \backslash \partial_{p}(\Gamma)$ is $F_{\sigma}$. For each infinite path in $\Gamma$ we can associate a set of extreme points, which is roughly the "points at infinity" of the path with respect to the $p$-Royden boundary. Our other main result in this paper is that the $p$-harmonic boundary is precisely the intersection of the extreme points of a certain subset of one-sided infinite paths in $\Gamma$.

The research for this paper was partially supported by PSC-CUNY grant 638730041 and I would like to thank them for their support.

\section{The $p$-Royden and $p$-harmonic boundaries}

Let $1<p \in \mathbf{R}$. In this section we construct the $p$-Royden and $p$-harmonic boundaries of $\Gamma$. For a more detailed discussion about this construction see Section 2.1 of [6]. Before we can give these definitions we need to define the space of $p$ Dirichlet finite functions on $V$. For any $S \subset V$, the outer boundary $\partial S$ of $S$ is the set of vertices in $V \backslash S$ with at least one neighbor in $S$. For a real-valued function $f$ on $S \cup \partial S$ we define the $p$-th power of the gradient, the $p$-Dirichlet sum, and the $p$-Laplacian of $x \in S$ by

$$
\begin{aligned}
|D f(x)|^{p} & =\sum_{y \in N_{x}}|f(y)-f(x)|^{p}, \quad I_{p}(f, S)=\sum_{x \in S}|D f(x)|^{p}, \\
\Delta_{p} f(x) & =\sum_{y \in N_{x}}|f(y)-f(x)|^{p-2}(f(y)-f(x)) .
\end{aligned}
$$

In the case $1<p<2$, we make the convention that $|f(y)-f(x)|^{p-2}(f(y)-f(x))=0$ if $f(y)=f(x)$. Let $S \subseteq V$. A function $f$ is said to be $p$-harmonic on $S$ if $\Delta_{p} f(x)=0$ for all $x \in S$. We shall say that $f$ is $p$-Dirichlet finite if $I_{p}(f, V)<\infty$. The set of all $p$-Dirichlet finite functions on $\Gamma$ will be denoted by $D_{p}(\Gamma)$. With respect to the following norm $D_{p}(\Gamma)$ is a reflexive Banach space,

$$
\|f\|_{D_{p}}=\left(I_{p}(f, V)+|f(o)|^{p}\right)^{1 / p},
$$

where $o$ is a fixed vertex of $\Gamma$ and $f \in D_{p}(\Gamma)$. We use $H D_{p}(\Gamma)$ to represent the set of $p$-harmonic functions on $V$ that are contained in $D_{p}(\Gamma)$. Let $\ell^{\infty}(\Gamma)$ denote the set of bounded functions on $V$ and let $\|f\|_{\infty}=\sup _{V}|f|$ for $f \in \ell^{\infty}(\Gamma)$. Set $B D_{p}(\Gamma)=D_{p}(\Gamma) \cap \ell^{\infty}(\Gamma)$. The set $B D_{p}(\Gamma)$ is a Banach space under the norm

$$
\|f\|_{B D_{p}}=\left(I_{p}(f, V)\right)^{1 / p}+\|f\|_{\infty}
$$

where $f \in B D_{p}(\Gamma)$. Let $B H D_{p}(\Gamma)$ be the set of bounded $p$-harmonic functions contained in $D_{p}(\Gamma)$. The space $B D_{p}(\Gamma)$ is also closed under the usual operations of scalar multiplication, addition and pointwise multiplication. Furthermore, $\|f g\|_{B D_{p}} \leq$ $\|f\|_{B D_{p}}\|g\|_{B D_{p}}$ for $f, g \in B D_{p}(\Gamma)$. Thus $B D_{p}(\Gamma)$ is a commutative Banach algebra. Let $C_{c}(\Gamma)$ be the set of functions on $V$ with finite support. Indicate the closure of $C_{c}(\Gamma)$ in $D_{p}(\Gamma)$ by ${\overline{C_{c}(\Gamma)}}_{D_{p}}$. Set $B\left({\overline{C_{c}(\Gamma)}}_{D_{p}}\right)={\overline{C_{c}(\Gamma)}}_{D_{p}} \cap \ell^{\infty}(\Gamma)$. Using the fact that the inequality $(a+b)^{1 / p} \leq a^{1 / p}+b^{1 / p}$ is true when $a, b \geq 0$ and $1<p \in \mathbf{R}$, we see immediately that $\|f\|_{D_{p}} \leq\|f\|_{B D_{p}}$. It now follows that $B\left({\overline{C_{c}(\Gamma)}}_{D_{p}}\right)$ is closed in $B D_{p}(\Gamma)$. 
Let $S p\left(B D_{p}(\Gamma)\right)$ denote the set of complex-valued characters on $B D_{p}(\Gamma)$, that is the nonzero ring homomorphisms from $B D_{p}(\Gamma)$ to $\mathbf{C}$. Then with respect to the weak $*$-topology, $S p\left(B D_{p}(\Gamma)\right)$ is a compact Hausdorff space. Given a topological space $X$, let $C(X)$ denote the ring of continuous functions on $X$ endowed with the sup-norm. The Gelfand transform defined by $\hat{f}(\chi)=\chi(f)$ yields a monomorphism of Banach algebras from $B D_{p}(\Gamma)$ into $C\left(S p\left(B D_{p}(\Gamma)\right)\right)$ with dense image. Furthermore the map $i: V \rightarrow S p\left(B D_{p}(\Gamma)\right)$ given by $(i(x))(f)=f(x)$ is an injection, and $i(V)$ is an open dense subset of $\operatorname{Sp}\left(B D_{p}(\Gamma)\right)$. For the rest of this paper, we shall write $f$ for $\hat{f}$, where $f \in B D_{p}(\Gamma)$. The $p$-Royden boundary of $\Gamma$, which we shall denote by $R_{p}(\Gamma)$, is the compact set $S p\left(B D_{p}(\Gamma)\right) \backslash i(V)$. The p-harmonic boundary of $\Gamma$ is the following subset of $R_{p}(\Gamma)$ :

$$
\partial_{p}(\Gamma):=\left\{\chi \in R_{p}(\Gamma) \mid \hat{f}(\chi)=0 \text { for all } f \in B\left({\overline{C_{c}(\Gamma)}}_{D_{p}}\right)\right\} .
$$

Let $S$ be an infinite subset of $V$ and let $A$ and $B$ be disjoint nonempty subsets of $S \cup \partial S$. The p-capacity of the condenser $(A, B, S)$ is defined by

$$
\operatorname{cap}_{p}(A, B, S)=\inf _{u} I_{p}(u),
$$

where the infimum is taken over all functions $u \in D_{p}(\Gamma)$ with $u=0$ on $A$ and $u=1$ on $B$. Such a function is called admissible. Set $\operatorname{cap}_{p}(A, B, S)=\infty$ if the set of admissible functions is empty.

Let $A$ be a finite subset of $S \cup \partial S$ and let $\left(U_{n}\right)$ be an exhaustion of $V$ by finite connected subsets such that $A \subset U_{1}$. We now define

$$
\operatorname{cap}_{p}(A, \infty, S)=\lim _{n \rightarrow \infty} \operatorname{cap}_{p}\left(A,(\partial S \cup S) \backslash U_{n}, S\right) .
$$

Since $\operatorname{cap}_{p}\left(A,(\partial S \cup S) \backslash U_{n}, S\right) \geq \operatorname{cap}_{p}\left(A,(\partial S \cup S) \backslash U_{n+1}, S\right)$, the above limit exists. We shall say that $S$ is p-hyperbolic if there exists a finite subset $A$ of $S \cup \partial S$ that satisfies $\operatorname{cap}_{p}(A, \infty, S)>0$. If $S$ is not $p$-hyperbolic, then it is said to be p-parabolic. An equivalent definition of $p$-parabolic is that $S$ is $p$-parabolic if and only if $1_{S} \in$ ${\overline{C_{c}\left(\Gamma_{S}\right)}}_{D_{p}}$, where $1_{S}$ is the constant function 1 on $S$ and $\Gamma_{S}$ the subgraph of $\Gamma$ induced by $S,[9$, Theorem 3.2]. We will define a graph $\Gamma$ to be $p$-hyperbolic ( $p$-parabolic) if its vertex set $V$ is $p$-hyperbolic ( $p$-parabolic). It was shown in [6, Proposition 4.2] that $\Gamma$ is $p$-parabolic if and only if $\partial_{p}(\Gamma)=\emptyset$. A useful property of $p$-hyperbolic graphs that we will use throughout this paper is the following $p$-Royden decomposition, see [6, Theorem 4.6] for a proof.

Theorem 2.1. ( $p$-Royden decomposition) Let $1<p \in \mathbf{R}$ and suppose $f \in$ $B D_{p}(\Gamma)$. Then there exists a unique $u \in B\left({\overline{C_{c}(\Gamma)}}_{D_{p}}\right)$ and a unique $h \in B H D_{p}(\Gamma)$ such that $f=u+h$.

Let $G$ be a finitely generated group. The Cayley graph of $G$ is an example of the type of graph we study in this paper. As was mentioned in Section 1 the first reduced $\ell^{p}$-cohomology space of $G$ vanishes if and only if the cardinality of the $p$ harmonic boundary of $G$ is one or zero. The reason for this is that the first reduced $\ell^{p}$-cohomology space of $G$ vanishes if and only if the only $p$-harmonic functions on $G$ that are contained in $D_{p}(G)$ are the constants, for a proof of this see the remark after Theorem 3.5 in [5]. Furthermore, [6, Theorem 2.5] tells us that there are nonconstant $p$-harmonic functions with finite $p$-Diriclet sum on a graph of bounded degree if and 
only if the cardinality of the $p$-harmonic boundary of the graph is greater than one. In section 7 of [6] the $p$-harmonic boundary is computed for several groups.

\section{Statement of main results}

In this section we will state our main results. In section 4 we will prove

Theorem 3.1. Let $1<p \in \mathbf{R}$ and let $\Gamma$ be a graph of bounded degree. The set $R_{p}(\Gamma) \backslash \partial_{p}(\Gamma)$ is $F_{\sigma}$.

Before we state our other main result we need to define the set of extreme points of a path in $\Gamma$. Let $P$ be the set of all one-sided infinite paths with no self-intersections in $\Gamma$. For a real-valued function $f$ on $V$ and a path $\gamma \in P$, the limit of $f$ as we follow $\gamma$ to infinity is given by $\lim _{n \rightarrow \infty} f\left(x_{n}\right)$, where $x_{0}, x_{1}, \ldots, x_{n}, \ldots$ is the vertex representation of the path $\gamma$. Sometimes we write $f(\gamma)=\lim _{n \rightarrow \infty} f\left(x_{n}\right)$ to indicate this limit. Let $\gamma \in P$ and denote by $V(\gamma)$ the set of vertices on $\gamma$. The closure of $i(V(\gamma))$ in $S p\left(B D_{p}(\Gamma)\right)$ will be indicated by $\bar{V}(\gamma)$. Recall that $S p\left(B D_{p}(\Gamma)\right)$ is endowed with the weak*-topology. Thus $\chi \in \bar{V}(\gamma)$ if and only if there exists a subsequence $\left(x_{n_{k}}\right)$ of $\left(x_{n}\right)$ such that $\lim _{k \rightarrow \infty} f\left(x_{n_{k}}\right)=\chi(f)$ for all $f \in B D_{p}(\Gamma)$. The extreme points of a path $\gamma$ is defined to be

$$
E(\gamma)=\bar{V}(\gamma) \cap R_{p}(\Gamma) .
$$

Let $f \in B\left({\overline{C_{c}(\Gamma)}}_{D_{p}}\right)$ and set $A_{f}=\{\gamma \in P \mid f(\gamma) \neq 0\}$. Set

$$
E_{f}=\overline{\left\{\cup_{\gamma} E(\gamma) \mid \gamma \in P \backslash A_{f}\right\}}
$$

In Section 5 we shall prove

Theorem 3.2. Let $1<p \in \mathbf{R}$ and let $\Gamma$ be a graph of bounded degree. Then

$$
\partial_{p}(\Gamma)=\cap_{f \in B\left({\overline{C_{c}(\Gamma)}}_{D_{p}}\right)} E_{f} .
$$

Let $1<p \in \mathbf{R}$. If $\Gamma$ is $p$-parabolic, then $\partial_{p}(\Gamma)=\emptyset$ and Theorem 3.1 is true. Also for the $p$-parabolic case, $1_{V} \in B\left({\overline{C_{c}(\Gamma)}}_{D_{p}}\right)$ by $\left[9\right.$, Theorem 3.2], where $1_{V}$ is the constant function one on $V$. Then $E_{1_{V}}=\emptyset$ and Theorem 3.2 follows. Thus for the rest of the paper we will assume $\Gamma$ is $p$-hyperbolic.

\section{Proof of Theorem 3.1}

In this section we will prove Theorem 3.1. We will start by giving some needed definitions and proving a comparison principle. A comparison principle for finite subsets of $V$ was proved in [2, Theorem 3.14]. Our proof follows theirs in spirit.

Let $f$ and $h$ be elements of $B D_{p}(\Gamma)$ and let $1<p \in \mathbf{R}$. Define

$$
\left\langle\Delta_{p} h, f\right\rangle:=\sum_{x \in V} \sum_{y \in N_{x}}|h(y)-h(x)|^{p-2}(h(y)-h(x))(f(y)-f(x)) .
$$

The sum exists since

$$
\sum_{x \in V} \sum_{y \in N_{x}}|| h(y)-\left.\left.h(x)\right|^{p-2}(h(y)-h(x))\right|^{q}=I_{p}(h, V)<\infty,
$$

where $\frac{1}{p}+\frac{1}{q}=1$. For notational convenience let

$$
T(h, f, x, y)=|h(y)-h(x)|^{p-2}(h(y)-h(x))(f(y)-f(x)) .
$$


In order to prove Theorem 3.1 we will need the following:

Lemma 4.1. (Comparison principle) Let $h_{1}, h_{2}$ be elements of $B H D_{p}(\Gamma)$ and suppose $h_{1}(x) \leq h_{2}(x)$ for all $x \in \partial_{p}(\Gamma)$. Then $h_{1} \leq h_{2}$ on $V$.

Proof. Define a function $f$ on $V$ by $f=\min \left\{h_{2}-h_{1}, 0\right\}$. Theorem 4.8 of [6] says $f \in B\left({\overline{C_{c}(\Gamma)}}_{D_{p}}\right)$ since $f=0$ on $\partial_{p}(\Gamma)$. By Lemma 4.6 of $[6]$ we have $\left\langle\Delta_{p} h_{1}, f\right\rangle=0$ and $\left\langle\Delta_{p} h_{2}, f\right\rangle=0$, which implies $\left\langle\Delta_{p} h_{1}-\Delta_{p} h_{2}, f\right\rangle=0$. Now set

$$
A=\left\{x \in V \mid h_{1}(x) \leq h_{2}(x)\right\}, \quad B=\left\{x \in V \mid h_{2}(x)<h_{1}(x)\right\},
$$

and for $a \in V$ let

$C_{a}=\left\{y \in V \mid y \in N_{a}\right.$ and $\left.h_{1}(y) \leq h_{2}(y)\right\}, D_{a}=\left\{y \in V \mid y \in N_{a}\right.$ and $\left.h_{2}(y)<h_{1}(y)\right\}$.

Now

$$
0=\sum_{x \in V} \sum_{y \in N_{x}}\left(T\left(h_{1}, f, x, y\right)-T\left(h_{2}, f, x, y\right)\right)=T_{1}+T_{2}+T_{3}
$$

where

$$
\begin{aligned}
& T_{1}=\sum_{x \in A} \sum_{y \in C_{x}}\left(T\left(h_{1}, f, x, y\right)-T\left(h_{2}, f, x, y\right)\right), \\
& T_{2}=\left(\sum_{x \in A} \sum_{y \in D_{x}}+\sum_{x \in B} \sum_{y \in C_{x}}\right)\left(T\left(h_{1}, f, x, y\right)-T\left(h_{2}, f, x, y\right)\right),
\end{aligned}
$$

and

$$
T_{3}=\sum_{x \in B} \sum_{y \in D_{x}}\left(T\left(h_{1}, f, x, y\right)-T\left(h_{2}, f, x, y\right)\right) .
$$

Since $f(x)=f(y)=0$ for $x \in A$ and $y \in C_{x}$ it follows that $T_{1}=0$. We now claim that $T_{3} \leq 0$. To see the claim let $a$ and $b$ be real numbers such that $a \neq b$. It follows from the inequality

$$
|a|^{p-2} a(a-b)>|b|^{p-2} b(a-b)
$$

that

$$
T\left(h_{1}, h_{1}-h_{2}, x, y\right) \geq T\left(h_{2}, h_{1}-h_{2}, x, y\right) .
$$

Equality occurs if and only if $\left(h_{1}-h_{2}\right)(x)=\left(h_{1}-h_{2}\right)(y)$. Now if $x \in B$ and $y \in D_{x}$, then $f(y)-f(x)=\left(h_{2}-h_{1}\right)(y)-\left(h_{2}-h_{1}\right)(x)$. Combining (4.2) with the fact $T\left(h_{k}, h_{1}-h_{2}, x, y\right)=-T\left(h_{k}, h_{2}-h_{1}, x, y\right)$, where $k=1$ or $k=2$, we obtain $T_{3} \leq 0$, which is our claim.

We now proceed to show that if there is a pair of vertices $x$ and $y$ that satisfy $x \in A, y \in D_{x}$ or $x \in B, y \in C_{x}$, then $T_{2}<0$. Suppose $x \in A$ and $y \in D_{x}$. Then $f(y)-f(x)=h_{2}(y)-h_{1}(y)<0$ and

$$
\begin{aligned}
T\left(h_{1}, f, x, y\right)-T\left(h_{2}, f, x, y\right)= & \left(h_{2}(y)-h_{1}(y)\right)\left(\left|h_{1}(y)-h_{1}(x)\right|^{p-2}\left(h_{1}(y)-h_{1}(x)\right)\right. \\
& \left.-\left|h_{2}(y)-h_{2}(x)\right|^{p-2}\left(h_{2}(y)-h_{2}(x)\right)\right) .
\end{aligned}
$$

Also $h_{1}(y)-h_{1}(x)>h_{2}(y)-h_{2}(x)$ because $h_{1}(y)-h_{2}(y)>0 \geq h_{1}(x)-h_{2}(x)$. So if $h_{2}(y) \geq h_{2}(x)$ we see that $T\left(h_{1}, f, x, y\right)-T\left(h_{2}, f, x, y\right)<0$ since $h_{1}(y)-h_{1}(x)>$ $h_{2}(y)-h_{2}(x)$. On the other hand if $h_{2}(y)<h_{2}(x)$ and $h_{1}(y)>h_{1}(x)$ we obtain

$$
\begin{aligned}
& T\left(h_{1}, f, x, y\right)-T\left(h_{2}, f, x, y\right) \\
& =\left(h_{2}(y)-h_{1}(y)\right)\left(\left|h_{1}(y)-h_{1}(x)\right|^{p-1}+\left|h_{2}(y)-h_{2}(x)\right|^{p-1}\right)<0
\end{aligned}
$$


since $\left|h_{2}(y)-h_{2}(x)\right|=-\left(h_{2}(y)-h_{2}(x)\right)$. The only other possibility is $h_{2}(y)<h_{2}(x)$ and $h_{1}(y) \leq h_{1}(x)$. If this is the case then $h_{2}(y)<h_{1}(y) \leq h_{1}(x) \leq h_{2}(x)$ due to $x \in A$ and $y \in D_{x}$. Consequently, $h_{2}(y)-h_{2}(x)<h_{1}(y)-h_{1}(x)$ and $h_{1}(x)-h_{1}(y)<$ $h_{2}(x)-h_{2}(y)$; hence, $\left|h_{1}(y)-h_{1}(x)\right|<\left|h_{2}(y)-h_{2}(x)\right|$. It now follows that

$$
\begin{aligned}
& T\left(h_{1}, f, x, y\right)-T\left(h_{2}, f, x, y\right) \\
& =\left(h_{2}(y)-h_{1}(y)\right)\left(\left|h_{2}(y)-h_{2}(x)\right|^{p-1}-\left|h_{1}(y)-h_{1}(x)\right|^{p-1}\right)<0 .
\end{aligned}
$$

A similar argument can be used to show that $T\left(h_{1}, f, x, y\right)-T\left(h_{2}, f, x, y\right)<0$ for each $x \in B$ and $y \in C_{x}$. Hence, if $x \in A, y \in D_{x}$ or $x \in B, y \in C_{x}$, then $T_{2}<0$. Since $T_{1}=0$ and $T_{3} \leq 0$, it follows from (4.1) that it must be the case $T_{2}=0$. Thus it is impossible to have a pair of vertices $x$ and $y$ with $x \in A, y \in D_{x}$ or $x \in B, y \in C_{x}$.

Now assume that $h_{1}(z)>h_{2}(z)$ for some $z \in V$. We claim that there exists vertices $x_{0}, y_{0}$ in $V$ for which $y_{0} \in N_{x_{0}}, h_{1}\left(x_{0}\right)>h_{2}\left(x_{0}\right)$ and $h_{1}\left(y_{0}\right) \leq h_{2}\left(y_{0}\right)$. To see the claim suppose $h_{1}=h_{2}$ on $\partial_{p}(\Gamma)$, then $h_{1}=h_{2}$ on $V$ by [6, Corollary 4.9]. So there exists an $x \in \partial_{p}(\Gamma)$ that satisfies $h_{1}(x)<h_{2}(x)$. Let $\left(x_{n}\right) \rightarrow x$ where $\left(x_{n}\right)$ is a sequence in $V$. Now there exists a term $x_{m}$ in this sequence such that $h_{1}\left(x_{m}\right)<h_{2}\left(x_{m}\right)$. Since $\Gamma$ is connected there is a path from $z$ to $x_{m}$. Thus there are vertices $x_{0}$ and $y_{0}$ on this path with $y_{0} \in N_{x_{0}}, h_{1}\left(x_{0}\right)>h_{2}\left(x_{0}\right)$, and $h_{1}\left(y_{0}\right)<h_{2}\left(y_{0}\right)$ because $h_{1}(z)>h_{2}(z)$ and $h_{1}\left(x_{m}\right)<h_{2}\left(x_{m}\right)$. Thus $x_{0} \in B$ and $y_{0} \in C_{x_{0}}$, a contradiction. Therefore, $h_{1}(z) \leq h_{2}(z)$ for all $z \in V$.

Proof of Theorem 3.1. Let $1<p \in \mathbf{R}$. Since $S p\left(B D_{p}(\Gamma)\right)$ is a normal space, there exists for each $x \in R_{p}(\Gamma)$ a sequence $\left(U_{j}(x)\right)$ of open sets containing $x$ such that $\bar{U}_{j+1}(x) \subseteq U_{j}(x)$. For each $j \in \mathbf{N}$ there exists a finite number of points $x_{j, k}, 1 \leq$ $k \leq N_{j}$ such that $U_{j}\left(x_{j, k}\right)$ cover $R_{p}(\Gamma)$. For notational simplicity we will denote $U_{j}\left(x_{j, k}\right)$ by $U_{j, k}$. Using Urysohn's lemma we can construct a continuous function $\phi_{j, k}$ with $\phi_{j, k}=2$ on $U_{j, k}$ and $\phi_{j, k}=-1$ on $S p\left(B D_{p}(\Gamma)\right) \backslash U_{j-1, k}$. By the density of $B D_{p}(\Gamma)$ in $C\left(S p\left(B D_{p}(\Gamma)\right)\right)$ there exists a $g \in B D_{p}(\Gamma)$ such that $\left|\phi_{j, k}-g\right|<\frac{1}{2}$. Set $f_{j, k}=\max (\min (1, g), 0)$, so $f_{j, k} \in B D_{p}(\Gamma), 0 \leq f_{j, k} \leq 1, f_{j, k}=1$ on $U_{j, k}$ and $f_{j, k}=0$ on $S p\left(B D_{p}(\Gamma)\right) \backslash U_{j-1, k}$. The $p$-Royden decomposition of $B D_{p}(\Gamma)$ yields a unique $p$-harmonic function $h_{j, k} \in B H D(\Gamma)$ and a unique $u_{j, k} \in B\left({\overline{C_{c}(\Gamma)}}_{D_{p}}\right)$ such that $f_{j, k}=u_{j, k}+h_{j, k}$. Because $u_{j, k}=0$ on $\partial_{p}(\Gamma)$ by $[6$, Theorem 4.8], we see that $f_{j, k}=h_{j, k}$ on $\partial_{p}(\Gamma)$. Now define

$$
R_{j, k}=\left\{x \in R_{p}(\Gamma) \cap \bar{U}_{j, k} \mid \lim _{x_{n} \rightarrow x} h_{j, k}\left(x_{n}\right)<f_{j, k}(x)=1\right\}
$$

where $\left(x_{n}\right)$ is a sequence in $V$. Observe that if $R_{j, k}$ is nonempty, then it only contains elements of $R_{p}(\Gamma) \backslash \partial_{p}(\Gamma)$.

Let $x \in R_{p}(\Gamma) \backslash \partial_{p}(\Gamma)$. We will now show that there exists $j, k \in \mathbf{N}$ such that $x \in R_{j, k}$. Since $x \notin \partial_{p}(\Gamma)$ there exists a $u \in B\left({\overline{C_{c}(\Gamma)}}_{D_{p}}\right)$ such that $u(x) \neq 0$. Since $B\left(\bar{C}_{c}(\Gamma)_{D_{p}}\right)$ is an ideal we may assume that $u \geq 0$ on $V$ and $u(x)>0$. Replacing $u$ by $u^{-1}(x) u$ if necessary we may assume that $u(x)=1$. Let $h \in B H D_{p}(\Gamma)$ that satisfies $h \geq 1$ on $V$. Set $f=u+h$, so $f \in B D_{p}(\Gamma)$ and $f=h$ on $\partial_{p}(\Gamma)$. Let $\left(x_{n}\right)$ be a sequence in $V$ that converges to $x$. Now $\lim _{n \rightarrow \infty} h\left(x_{n}\right)<f(x)$. Because $f$ is continuous we can find an open set $U_{j, k}$ that contains $x$ and satisfies

$$
m=\inf _{U_{j-1, k} \cap R_{p}(\Gamma)} f>\lim _{n \rightarrow \infty} h\left(x_{n}\right) .
$$


It now follows

$$
f_{j, k} \leq \frac{f}{m} \text { on } R_{p}(\Gamma)
$$

which implies that $h_{j, k} \leq \frac{h}{m}$ on $\partial_{p}(\Gamma)$. An appeal to the comparison principle gives us

$$
\lim _{n \rightarrow \infty} h_{j, k}\left(x_{n}\right) \leq \frac{1}{m} \lim _{n \rightarrow \infty} h\left(x_{n}\right)<1=f_{j, k}(x),
$$

hence $x \in R_{j, k}$. Furthermore,

$$
R_{j, k}=\bigcup_{i=1}^{\infty}\left(R_{p}(\Gamma) \cap \bar{U}_{j, k} \cap\left\{\overline{y \in V \mid h_{j, k}(y)<1-1 / i}\right\}\right) .
$$

Thus $R_{j, k}$ is a countable union of compact sets. Theorem 3.1 now follows because

$$
R_{p}(\Gamma) \backslash \partial_{p}(\Gamma)=\bigcup_{j=1}^{\infty} \bigcup_{k=1}^{N_{j}} R_{j, k} .
$$

\section{Proof of Theorem 3.2}

Before we prove Theorem 3.2 we need to state some definitions and prove several preliminary results.

Fix a real number $p>1$. Recall that $E$ denotes the edge set of a graph $\Gamma$. Denote by $\mathcal{F}(E)$ the set of all real-valued functions on $E$ and let $\mathcal{F}^{+}(E)$ be the subset of $\mathcal{F}(E)$ that consists of all nonnegative functions. For $\omega \in \mathcal{F}(E)$ set

$$
\mathcal{E}_{p}(\omega)=\sum_{e \in E}|\omega(e)|^{p} .
$$

The edge set of a path $\gamma$ in $\Gamma$ will be denoted by $E d(\gamma)$, remember $E(\gamma)$ represents the extreme points of $\gamma$. Let $Q$ be a set of paths in $\Gamma$, denote by $\mathcal{A}(Q)$ the set of all $\omega \in \mathcal{F}^{+}(E)$ that satisfy $\mathcal{E}_{p}(\omega)<\infty$ and $\sum_{e \in E d(\gamma)} \omega(e) \geq 1$ for each $\gamma \in Q$. The extremal length of order $p$ for $Q$ is defined by

$$
\lambda_{p}(Q)^{-1}=\inf \left\{\mathcal{E}_{p}(\omega) \mid \omega \in \mathcal{A}(Q)\right\} .
$$

A variation of the next lemma was proved for the case $p=2$ in [7, Lemma 6.13]. In the $p=2$ case the conclusion of the lemma is stronger in that $g$ belongs to $\bar{C}_{c}(\Gamma){ }_{D_{2}}$ instead of the larger space $D_{2}(\Gamma)$.

Lemma 5.1. Let $K$ be a compact subset of $R_{p}(\Gamma)$ with $K \cap \partial_{p}(\Gamma)=\emptyset$. Then there exists a function $g \in D_{p}(\Gamma)$ that satisfies $g=\infty$ on $K$ and $g=0$ on $\partial_{p}(\Gamma)$.

Proof. By Urysohn's lemma there exists an $f \in C\left(S p\left(B D_{p}(\Gamma)\right)\right)$ that satisfies the following: $0 \leq f \leq 1, f=1$ on $K$ and $f=0$ on $\partial_{p}(\Gamma)$. Using the argument from the first paragraph of the proof of Theorem 3.1 we may and do assume $f \in B D_{p}(\Gamma)$.

Let $\left(U_{n}\right)$ be an exhaustion of $V$ by finite connected subsets. Applying Theorem 3.1 of [2] yields a function $h_{n}$ that is $p$-harmonic on $U_{n}$ and equals $f$ on $V \backslash U_{n}$. It follows from the minimizer property of $p$-harmonic functions on $U_{n}$ that $I_{p}\left(h_{n}, V\right) \leq I_{p}(f, V)$. Hence, $h_{n} \in B D_{p}(\Gamma)$ for each $n \in \mathbf{N}$. Also, $h_{n}=0$ on $\partial_{p}(\Gamma), h_{n}=1$ on $K$ and $0 \leq h_{n} \leq 1$ for each $n$. By passing to a subsequence if necessary, we may assume that $\left(h_{n}\right)$ converges pointwise to a function $h$ because $\overline{\left\{h_{n}(x) \mid n \in \mathbf{N}\right\}}$ is compact for each $x \in V$. By Lemma 3.2 of [2], $h$ is $p$-harmonic on $V$. Since the sequence $\left(I_{p}\left(h_{n}, V\right)\right)$ is bounded, Theorem 1.6 on page 177 of [8] says 
that by passing to a subsequence if necessary, we may assume that $\left(h_{n}\right)$ converges weakly to a function $\bar{h} \in D_{p}(\Gamma)$. Because evaluation by $x \in V$ is a continuous linear functional on $D_{p}(\Gamma)$, we have that $h_{n}(x) \rightarrow \bar{h}(x)$ for each $x \in V$. Thus $h=\bar{h}$ and $h \in B D_{p}(\Gamma)$. It follows from [6, Corollary 4.9] that $h=0$ on $V$, due to that fact $h=0$ on $\partial_{p}(\Gamma)$.

Since $h_{n} \rightarrow h$ pointwise on $U_{k}$ for $k \in \mathbf{N}$, it follows $I_{p}\left(h_{n}, U_{k}\right) \rightarrow I_{p}\left(h, U_{k}\right)=0$ for each $k$. Consequently, $I_{p}\left(h_{n}, V\right) \rightarrow 0$. By taking a subsequence if necessary, we may assume that $\left\|h_{n}\right\|_{D_{p}} \leq 2^{-n}$. Let $\epsilon>0$ be given and for $m \in \mathbf{N}$, let $g_{m}=\sum_{k=1}^{m} h_{k}$. There exists $N \in \mathbf{N}$ such that $2^{-N}<\epsilon$. For $m, n \in \mathbf{N}$ with $m>n \geq N$ we see that

$$
\left\|g_{m}-g_{n}\right\|_{D_{p}}=\left\|\sum_{k=n+1}^{m} h_{k}\right\|_{D_{p}} \leq \sum_{k=n+1}^{m} 2^{-k}<2^{-n}<\epsilon .
$$

Hence, the Cauchy sequence $\left(g_{m}\right)$ converges to $g=\sum_{k=1}^{\infty} h_{k}$ in the $D_{p}$-norm. Thus $g \in D_{p}(\Gamma)$. For $x \in K, g_{m}(x)=m$, so $g(x)=\infty$; also $g=g_{m}=0$ on $\partial_{p}(\Gamma)$. The proof of the lemma is complete.

The next result was shown to be true for the case $p=2$ in [7, Theorem 6.16]. Our proof is essentially the same, and we include it for completeness.

Lemma 5.2. Let $P$ be a family of one-sided infinite paths in $\Gamma$ and let

$$
K=\overline{\cup_{\gamma \in P} E(\gamma)}
$$

If $K$ is disjoint from $\partial_{p}(\Gamma)$, then $\lambda_{p}(P)=\infty$.

Proof. By Lemma 5.1 there exists a $g \in D_{p}(\Gamma)$ such that $g=\infty$ on $K$ and $g=0$ on $\partial_{p}(\Gamma)$. Let $\gamma \in P$ and let $x_{1}, x_{2}, x_{3}, \ldots$ be the vertex representation of $\gamma$. Since $E(\gamma) \subseteq K$ we have that $g(\gamma)=\lim _{k \rightarrow \infty} g\left(x_{k}\right)=\infty$. Thus

$$
\sum_{k=1}^{\infty}\left|g\left(x_{k}\right)-g\left(x_{k+1}\right)\right| \geq \lim _{k \rightarrow \infty}\left(g\left(x_{k}\right)-g\left(x_{1}\right)\right)=\infty .
$$

By [3, Lemma 2.3] we obtain $\lambda_{p}(P)=\infty$.

A connected infinite subset $D$ of $V$ with $\partial D \neq \emptyset$ is defined to be $D_{p}$-massive if there exists a $p$-harmonic function $u$ on $D$ that satisfies the following: $0 \leq u \leq$ $1, u=0$ on $\partial D, \sup _{D} u=1$ and $I_{p}(u, D)<\infty$. The function $u$ is known as an inner potential of $D$.

Proposition 5.3. Let $D$ be a $D_{p}$-massive subset, with inner potential $u$, of $V$. Denote by $P_{D}$ the set of all one-sided infinite paths contained in $D \cup \partial D$. Then $\lambda_{p}\left(P_{D}\right)<\infty$.

Proof. Let $a \in D$ and let $P_{a}$ be the set of all paths in $P_{D}$ with initial point $a$. If $\lambda_{p}\left(P_{a}\right)<\infty$, then $\lambda_{p}\left(P_{D}\right)<\infty$ by [3, Lemma 2.1]. Let $\left(B_{n}\right)$ be an exhaustion of $V$ by finite connected subsets of $V$ such that $B_{1} \cap \partial D \neq \emptyset$. Pick an $a \in B_{1} \cap \partial D$. By combining Theorem 2.1 and Theorem 2.4 of [4] we see that $\lambda_{p}\left(P_{a}\right)<\infty$ if and only if $\operatorname{cap}_{p}(\{a\}, \infty, D)>0$. Thus to finish the proof we need to show $\operatorname{cap}_{p}(\{a\}, \infty, D)>0$, which we now proceed to do.

Choose admissible functions $\omega_{k}, k \geq 2$, for condensers $\left(\{a\},(D \cup \partial D) \backslash B_{k}, D\right)$ such that

$$
I_{p}\left(\omega_{k}, D \cap B_{k}\right) \leq \operatorname{cap}_{p}\left(\{a\},(D \cup \partial D) \backslash B_{k}, D\right)+\frac{1}{k} .
$$


Replacing all values of $\omega_{k}(x)$ on $D \cap B_{k}$ for which $\omega_{k}(x)<0$ by 0 and replacing all values of $\omega_{k}(x)$ on $D \cap B_{k}$ for which $\omega_{k}(x)>1$ by 1 decreases the value of $I_{p}\left(\omega_{k}, D \cap B_{k}\right)$. Thus we may and do assume $0 \leq \omega_{k} \leq 1$ on $D \cap B_{k}$. Theorem 3.11 of [2] tells us that there exists a unique $p$-harmonic function $v_{2}$ on $D \cap B_{2}$ such that $v_{2}=\omega_{2}$ on $\partial\left(D \cap B_{2}\right)$. Extend $v_{2}$ to all of $D$ by setting $v_{2}=1$ on $D \backslash B_{2}$. By the minimizing property of $p$-harmonic functions,

$$
I_{p}\left(v_{2}, D \cap B_{2}\right) \leq I_{p}\left(\omega_{2}, D \cap B_{2}\right) .
$$

Since $u$ is $p$-harmonic on $D$ and $u(x) \leq v_{2}(x)$ for all $x \in \partial\left(D \cap B_{2}\right), u \leq v_{2}$ on $D \cap B_{2}$ by [2, Theorem 3.14]. Pick $\omega_{3}$. The set $A=\left\{x \in D \mid \omega_{3}(x)>v_{2}(x)\right\}$ is a subset of $D \cap B_{2}$. If $A \neq \emptyset$, redefine $\omega_{3}$ by setting $\omega_{3}=v_{2}$ on $A$. The redefined $\omega_{3}$ decreases $I_{p}\left(\omega_{3}, D \cap B_{3}\right)$, so (5.1) remains true. By continuing as above, we obtain a decreasing sequence of functions $\left(v_{k}\right)$ such that $v_{k}$ is $p$-harmonic on $B \cap B_{k}, v_{k} \geq u$, and

$$
I_{p}\left(v_{k}, D \cap B_{k}\right) \leq I_{p}\left(\omega_{k}, D \cap B_{k}\right) .
$$

Now assume that $\operatorname{cap}_{p}\left(\{a\},(D \cup \partial D) \backslash B_{k}, D\right) \rightarrow 0$. Then $I_{p}\left(v_{k}, D \cap B_{k}\right) \rightarrow 0$. Since $v_{k} \geq u$ and $\sup _{D} u=1$, it must be the case that $\left(v_{k}\right) \rightarrow 1_{D}$, the constant function 1 on $D$. This is a contradiction because $\left(v_{k}\right)$ is a decreasing sequence of functions, $0 \leq v_{2} \leq 1$ and $v_{2} \neq 1$. Thus, $\operatorname{cap}_{p}(\{a\}, \infty, D)>0$ and the proof of the proposition is complete.

Our next result is $[7$, Theorem 6.18$]$ for the case $p=2$. We give a different proof of the result.

Lemma 5.4. Let $P$ be the family of all one-sided infinite paths in $\Gamma$ and let $P_{\infty} \subseteq P$ be any subfamily with $\lambda_{p}\left(P_{\infty}\right)=\infty$. Then

$$
\partial_{p}(\Gamma) \subseteq \overline{\left\{\cup_{\gamma} E(\gamma) \mid \gamma \in P \backslash P_{\infty}\right\}} .
$$

Proof. Set $K=\overline{\left\{\cup_{\gamma} E(\gamma) \mid \gamma \in P \backslash P_{\infty}\right\}}$. Since our standing assumption is that $\Gamma$ is $p$-hyperbolic, it follows from [4, theorem 2.1] that $\lambda_{p}(P)<\infty$. By [3, Lemma 2.2], $\lambda_{p}\left(P \backslash P_{\infty}\right)<\infty$. Lemma 5.2 tells us $K \cap \partial_{p}(\Gamma) \neq \emptyset$. For purposes of contradiction, assume that there exists a $\chi \in \partial_{p}(\Gamma)$ for which $\chi \notin K$. By Urysohn's lemma there exists a continuous function $f$ on $S p\left(B D_{p}(\Gamma)\right)$ that satisfies the following: $0 \leq f \leq$ $1, f(\chi)=1$ and $f=0$ on $K \cap \partial_{p}(\Gamma)$. By density of $B D_{p}(\Gamma)$ in $C\left(S p\left(B D_{p}(\Gamma)\right)\right)$ we assume $f \in B D_{p}(\Gamma)$. The $p$-Royden decomposition for $B D_{p}(\Gamma)$ yields a unique $p$ harmonic function $h$ on $V$ and a unique $g \in B\left(\bar{C}_{c}(\Gamma)_{D_{p}}\right)$ such that $f=g+h$. Theorem 4.8 of [6] shows that $g=0$ on $\partial_{p}(\Gamma)$. Combining this fact with the maximum principe ([6, Theorem 4.7]) it follows that $0<h<1$ on $V, h(\chi)=1$ and $h=0$ on $\partial_{p}(\Gamma) \cap K$. Let

$$
A=\{x \in V \mid h(x)>1-\epsilon\},
$$

where $0<\epsilon<1$. Let $B$ be a component of $A$. The set $B$ is $D_{p}$-massive, see the proof of [6, Proposition 4.12] for a proof of this fact. Let $P_{A}$ be the family of all one-sided infinite paths in $A$, and let $P_{B}$ consist of all one-sided infinite paths in $B$. Since $B$ is a $D_{p}$-massive set, $\lambda_{p}\left(P_{B}\right)<\infty$ by Proposition 5.3. It now follows from [3, Lemma 2.1] that $\lambda_{p}\left(P_{A}\right)<\infty$. Set

$$
K_{1}=\overline{\left\{\cup_{\gamma} E(\gamma) \mid \gamma \in P_{A} \backslash P_{\infty}\right\}} .
$$

Another appeal to Lemma 5.2 shows $K_{1} \cap \partial_{p}(\Gamma) \neq \emptyset$, because $\lambda_{p}\left(P_{A} \backslash P_{\infty}\right)<\infty$. Furthermore, $h=0$ on $K_{1} \cap \partial_{p}(\Gamma)$ since $K_{1} \cap \partial_{p}(\Gamma) \subseteq K \cap \partial_{p}(\Gamma)$. However, $h(\gamma) \geq 1-\epsilon$ 
for all $\gamma \in P_{A}$. Thus we obtain the contradiction $h(x) \geq 1-\epsilon$ for all $x \in K_{1}$. Therefore, $\partial_{p}(\Gamma) \subseteq K$, as desired.

Proof of Theorem 3.2. Let $f \in B\left({\overline{C_{c}(\Gamma)}}_{D_{p}}\right)$ and let $a \in V$. Denote by $P_{a}$ the set of all one-sided infinite paths in $\Gamma$ with initial point $a$. Set

$$
A_{a, f}=\left\{\gamma \in P_{a} \mid f(\gamma) \neq 0\right\} .
$$

By [3, Theorem 3.3], $\lambda_{p}\left(A_{a, f}\right)=\infty$. Also, [3, Lemma 2.2] tells us $\lambda_{p}\left(A_{f}\right)=$ $\lambda_{p}\left(\cup_{a \in V} A_{a, f}\right)=\infty$. The definition of $A_{f}$ above and $E_{f}$ below were given in Section 3. Now Proposition 5.4 says that

$$
\partial_{p}(\Gamma) \subseteq E_{f}
$$

For notational convenience set $F=\cap_{f} E_{f}$, where $f$ runs through $B\left({\overline{C_{c}(\Gamma)}}_{D_{p}}\right)$. Thus, $\partial_{p}(\Gamma) \subseteq F$. We now proceed to prove the reverse inclusion. Suppose there exists a $\chi \in F$ for which $\chi \notin \partial_{p}(\Gamma)$. By [6, Theorem 4.8] we obtain an $f \in B\left(\bar{C}_{c}(\Gamma)_{D_{p}}\right)$ for which $\chi(f) \neq 0$. Let $\alpha \sim x_{0}, x_{1}, \ldots, x_{n}, \ldots$ be a one-sided path with $\chi \in \bar{V}(\alpha)$. Because $\chi(f) \neq 0$, there is a subsequence $\left(x_{n_{k}}\right)$ of $\left(x_{n}\right)$ that satisfies $\lim _{k \rightarrow \infty} f\left(x_{n_{k}}\right) \neq$ 0 . Thus $f(\alpha) \neq 0$ and has a result $\alpha \in A_{f}$. Hence $\chi \notin\left\{\cup_{\gamma} E(\gamma) \mid \gamma \in P \backslash A_{f}\right\}$. We are assuming $\chi \in E_{f}$, so it must be the case that there is a sequence $\left(\chi_{n}\right)$ in $\left\{\cup_{\gamma} E(\gamma) \mid \gamma \in P \backslash A_{f}\right\}$ with $\left(\chi_{n}\right) \rightarrow \chi$. Since $f(\gamma)=0$ for each $\gamma \in P \backslash A_{f}$ it follows immediately that $\chi_{n}(f)=0$ for each $n \in \mathbf{N}$. This implies $\chi(f)=0$, contradicting our assumption $\chi(f) \neq 0$. Therefore, $F \subseteq \partial_{p}(\Gamma)$. The proof of Theorem 3.2 is now complete.

\section{References}

[1] Gromov, M.: Asymptotic invariants of infinite groups. - In: Geometric group theory, vol. 2 (Sussex, 1991), London Math. Soc. Lecture Note Ser. 182, Cambridge Univ. Press, Cambridge, 1993, 1-295.

[2] Holopainen, I., and P.M. SoArdi: p-harmonic functions on graphs and manifolds. Manuscripta Math. 94:1, 1997, 95-110.

[3] Kayano, T., and M. Yamasaki: Boundary limit of discrete Dirichlet potentials. - Hiroshima Math. J. 14:2, 1984, 401-406.

[4] Nakamura, T., and M. Yamasaki: Generalized extremal length of an infinite network. Hiroshima Math. J. 6:1, 1976, 95-111.

[5] Puls, M. J.: The first $L^{p}$-cohomology of some finitely generated groups and $p$-harmonic functions. - J. Funct. Anal. 237:2, 2006, 391-401.

[6] Puls, M.: Graphs of bounded degree and the p-harmonic boundary. - Pacific J. Math. 248:2, 2010, 429-452.

[7] SoArdi, P. M.: Potential theory on infinite networks. - Lecture Notes in Math. 1590, SpringerVerlag, Berlin, 1994.

[8] TAYLOR, A.E., and D. C. LAY: Introduction to functional analysis. - Robert E. Krieger Publishing Co. Inc., Melbourne, FL, second edition, 1986.

[9] Yamasaki, M.: Parabolic and hyperbolic infinite networks. - Hiroshima Math. J. 7:1, 1977, $135-146$

Received 1 December 2010

Revised received 15 February 2011 